\title{
Influence of the Static Pre-Stress in Micro-Viscothermoelastic Resonators Based on Dual-Phase-Lagging Heat Conduction
}

\author{
Najat A. Alghamdi $\mathbb{D}^{1}$ and Hamdy M. Youssef $\mathbb{D}^{2,3}$ \\ ${ }^{1}$ Mathematics Department, Faculty of Applied Science, Umm Al-Qura University, Makkah, Saudi Arabia \\ ${ }^{2}$ Department of Mathematics, Faculty of Education, Alexandria University, Alexandria, Egypt \\ ${ }^{3}$ Mechanical Engineering Department-College of Engineering and Islamic Architecture, Umm Al-Qura University, Makkah, \\ Saudi Arabia \\ Correspondence should be addressed to Najat A. Alghamdi; najatalghamdi@gmail.com
}

Received 29 January 2020; Revised 10 April 2020; Accepted 25 June 2020; Published 27 July 2020

Academic Editor: Fumihiro Ashida

Copyright (C) 2020 Najat A. Alghamdi and Hamdy M. Youssef. This is an open access article distributed under the Creative Commons Attribution License, which permits unrestricted use, distribution, and reproduction in any medium, provided the original work is properly cited.

\begin{abstract}
Thermal and mechanical relaxation times play vital roles in the values of the quality factor of micro/nanoresonators. They can control the energy dissipation across the coupling of mechanical and thermal behavior. In this paper, we introduce an analytical model that considers a pre-stress in a micro-viscothermoelastic resonator to modify the thermal and mechanical relaxation times and thus higher the quality factor. The impacts of length scale and static pre-stress on the quality factor have been discussed. The model expects that significant improvement in terms of quality factors is possible by tuning the pre-stress and the thermal and mechanical relaxation times parameters, and the isothermal value of frequency have significant effects on the thermal quality factor of the resonators.
\end{abstract}

\section{Introduction}

Many applications based on microelectromechanical resonators are essential in different fields, such as mechanical signal processing, scanning probe microscopes, and ultrasensitive mass detection. The most critical parameter of micro-viscothermoelastic resonators is the quality factor $Q$. Higher $Q$ factor indicates less energy. The study of the energy dissipation mechanism is significant for the improvement of the design of micro/nanoelectromechanical resonators [1-4].

Zener [5-7] is the first who introduced the Q-factor in thermoelastic dissipation, and he gave an approximate analytical form of it. He has studied the thermoelastic damping in beams by treating the viscoelastic material. Many research studies have been carried out on the thermoelastic damping in which the classical theory of thermoelasticity based on the Fourier heat law of heat conduction has been used [8]. Lifshitz and Roukes [9] introduced an exact expression for thermoelastic damping. Sun et al. [10] study thermoelastic damping of a beam resonator based on a non-Fourier heat equation. Sharma and Sharma [11] studied damping in micro-scale circular plate resonators using the Lord-Shulman theory of generalized thermoelasticity theory $(L-S)$.

Tzou $[12,13]$ proposed a mathematical model to study heat conduction known as dual-phase-lag (DPL). This model established the temperature gradient and heat flux. Many scientists used this model in heat transfer problems [8, 14], physical systems [15-19], and thermoelastic damping vibration [20, 21]. Guo et al. [22, 23] studied the thermoelastic damping theory of micro- and nanomechanical resonators by using the DPL model.

The study of viscoelastic materials has become essential in mechanics. Biot $[24,25]$ discussed the theory of viscothermoelasticity in thermodynamics. A thermoviscoelasticity model of polymers at finite strains was derived by Drozdov [26]. A new model of thermoviscoelasticity for isotropic media was established by Ezzat and El-Karmany [27].

As the size of a flexural resonator is reduced, its natural frequency increases and thermoelastic damping also 
increases in the process. The natural frequency of beams can also be changed by the application of an axial force $[3,28]$. A compressive force decreases in the natural frequency, whereas a tensile force increases in the natural frequency [8]. Experimental results have been utilizing the frequency change to tune resonators. Furthermore, these experiments also suggest an increase in the $Q$-factor with the application of tensile stress [3, 29].

In the present study, we present an analytical model that considers a pre-stress in a micro-viscothermoelastic resonator to quantify the quality factor $Q$. We also analyze the results with respect to the resonator size and pre-stress and then compare the analytical results with the experimental results available in the literature.

\section{Basic Equations and Model Formulation}

According to Zener's model of a linear anelastic solid, the stress equation takes the following form [6]:

$$
\sigma=\sigma_{0}+\sigma_{1} e^{i \omega t}
$$

where $\sigma_{0}$ is the static pre-stress, and the second part represents the dynamic-stress due to the ensuing vibration and $\omega$ is the natural frequency. $[6,8]$

The resultant strain for a thermoelastic solid is given as

$$
\varepsilon=\frac{\sigma_{0}}{E_{R}}-\frac{\sigma_{0}}{E_{2}} e^{\left(-t / \tau_{\sigma}\right)}+\varepsilon_{1} e^{i \omega t}
$$

Making derivatives of equations (1) and (2) with respect to time and inserting them into the equation for a thermoelastic solid are given as follows:

$$
\sigma+\tau_{\varepsilon} \dot{\sigma}=E_{R}\left(\varepsilon+\tau_{\sigma} \dot{\varepsilon}\right)
$$

where $E_{R}$ is relaxed elastic modulus and $\tau_{\varepsilon}$ and $\tau_{\sigma}$ are constants strain and constant stress relaxation time constants; we obtain

$$
\begin{aligned}
\sigma_{0}+\sigma_{1} e^{i \omega t}+\tau_{\varepsilon}\left(i \omega \sigma_{1} e^{i \omega t}\right)= & E_{R}\left[\frac{\sigma_{0}}{E_{R}}-\frac{\sigma_{0}}{E_{2}} e^{\left(-t / \tau_{\sigma}\right)}+\varepsilon_{1} e^{i \omega t}\right. \\
& \left.+\tau_{\sigma}\left(\frac{1}{\tau_{\sigma}} \frac{\sigma_{0}}{E_{2}} e^{\left(-t / \tau_{\sigma}\right)}+i \omega \varepsilon_{1} e^{i \omega t}\right)\right] .
\end{aligned}
$$

All the terms due to static stress $\sigma_{0}$ get canceled from both sides. That results in static stress independence of Zener's thermoelastic solid model. This model assumes no contribution of a stress field to the thermal relaxation time in solids. Thus, we decided to follow Lifshitz-Roukes' approach to the exact solution for a pre-stressed beam resonator. The constitutive relationship for Euler-Bernoulli's beam with dimensions length $L$, width $b$, and depth $h$ along the $x, y$, and $z$-axes, respectively, the moment of inertia $I$, and applied axial force $F$ and flexural displacement $w(x, y, z, t)$ can be written as follows:

$$
\sigma_{x x}=\sigma_{0}-E y \frac{\partial^{2} w}{\partial x^{2}}-E \alpha_{T} \theta
$$

where $\sigma_{0}=(F / b h)$ is the stress due to the applied axial force, $E$ is Young's Modulus, and $\alpha_{T}$ is the coefficient of thermal expansion. The equilibrium temperature of the beam is $T_{0}$, and the ensuing vibration will result in a temperature field $T=T_{0}+\theta$.

By deriving the equation of motion for flexural vibration, we obtain

$$
E I \frac{\partial^{4} w}{\partial x^{4}}-F \frac{\partial^{2} w}{\partial x^{2}}+E \alpha_{T} \frac{\partial^{2} I_{T}}{\partial x^{2}}+\rho A \frac{\partial^{2} w}{\partial t^{2}}=0,
$$

where

$$
\begin{aligned}
I & =\int_{(-b / 2)}^{(b / 2)} \int_{(-h / 2)}^{(h / 2)} y^{2} \mathrm{~d} y \mathrm{~d} z=\frac{\mathrm{bh}^{3}}{12}, \\
I_{T} & =\int_{(-b / 2)}^{(b / 2)} \int_{(-h / 2)}^{(h / 2)} y \theta \mathrm{d} y \mathrm{~d} z .
\end{aligned}
$$

The dual-phase-lag heat equation takes the form

$$
K\left(1+\tau_{T} \frac{\partial}{\partial t}\right) \nabla^{2} \theta=\left(\frac{\partial}{\partial t}+\tau_{q} \frac{\partial^{2}}{\partial t^{2}}\right)\left(\rho C_{\nu} \theta+\frac{E \alpha_{T} T_{0}}{(1-2 \nu)} \varepsilon\right),
$$

where $K$ is the thermal conductivity, $v$ is Poisson's ratio, $C_{v}$ is the specific heat at constant strain, and $\tau_{q}$ and $\tau_{T}$ are the thermal relaxation of the temperature and its gradient, respectively.

The volumetric deformations are as follows:

$$
\varepsilon=\varepsilon_{\mathrm{xx}}+\varepsilon_{\mathrm{yy}}+\varepsilon_{\mathrm{zz}}
$$

where

$$
\begin{aligned}
& E \varepsilon_{\mathrm{xx}}=\sigma_{0}-y E \frac{\partial^{2} w}{\partial x^{2}} \\
& E \varepsilon_{\mathrm{yy}}=E \varepsilon_{\mathrm{zz}}=-v \sigma_{0}+v y E \frac{\partial^{2} w}{\partial x^{2}}+(1+v) \alpha_{T} E \theta .
\end{aligned}
$$

Then, we have

$$
E \varepsilon=(1-2 v) \sigma_{0}-(1-2 v) y E \frac{\partial^{2} w}{\partial x^{2}}+2(1+v) \alpha_{T} E \theta .
$$

Substituting from equation (11) into equation (9), we obtain

$$
\begin{aligned}
\left(1+\tau_{T} \frac{\partial}{\partial t}\right) \nabla^{2} \theta= & \eta\left(\frac{\partial}{\partial t}+\tau_{q} \frac{\partial^{2}}{\partial t^{2}}\right) \theta+\frac{\alpha_{T} T_{0}}{K}\left(\frac{\partial}{\partial t}+\tau_{q} \frac{\partial^{2}}{\partial t^{2}}\right) \\
& \cdot\left[2 \frac{(1+v) \alpha_{T}}{(1-2 v)} E \theta-y E \frac{\partial^{2} w}{\partial x^{2}}\right],
\end{aligned}
$$

which gives

$$
\begin{gathered}
\left(1+\tau_{T} \frac{\partial}{\partial t}\right) \nabla^{2} \theta+\frac{\alpha_{T} T_{0} E}{K} y\left(\frac{\partial}{\partial t}+\tau_{q} \frac{\partial^{2}}{\partial t^{2}}\right) \frac{\partial^{2} w}{\partial x^{2}} \\
=\left(\frac{\partial}{\partial t}+\tau_{q} \frac{\partial^{2}}{\partial t^{2}}\right)\left(\eta+\frac{2 E T_{0} \alpha_{T}^{2}(1+v)}{K(1-2 \nu)}\right) \theta,
\end{gathered}
$$


where $\eta=\left(\rho C_{v} / K\right)$.

For viscothermoelastic materials, we consider Young's modulus in the form $[24,27]$

$$
E=E_{0}\left(1+E_{1} \frac{\partial}{\partial t}\right)
$$

where $E_{0}$ is Young's modulus value for the usual case, while $E_{1}$ is the mechanical relaxation time (relaxation time means the return of a perturbed system into equilibrium or the time between repeating mechanical waves).

Hence, equation (14) takes the form

$$
\begin{gathered}
\left(1+\tau_{T} \frac{\partial}{\partial t}\right) \nabla^{2} \theta+\frac{\Delta_{E}}{\alpha_{T}}\left(1+E_{1} \frac{\partial}{\partial t}\right)\left(\frac{\partial}{\partial t}+\tau_{q} \frac{\partial^{2}}{\partial t^{2}}\right) y \frac{\partial^{2} w}{\partial x^{2}} \\
=\left(\frac{\partial}{\partial t}+\tau_{q} \frac{\partial^{2}}{\partial t^{2}}\right)\left(\eta+2 \Delta_{E} \frac{(1+v)}{(1-2 v)}\left(1+E_{1} \frac{\partial}{\partial t}\right)\right) \theta,
\end{gathered}
$$

where $\Delta_{E}=\left(T_{0} \alpha_{T}^{2} E_{0} / K\right)$.

Because no gradient exists in the $z$-direction, then $\nabla^{2} \theta=\left(\partial^{2} \theta / \partial y^{2}\right)$; therefore, we have

$$
\begin{gathered}
\left(1+\tau_{T} \frac{\partial}{\partial t}\right) \frac{\partial^{2} \theta}{\partial y^{2}}+\frac{\Delta_{E}}{\alpha_{T}} y\left(1+E_{1} \frac{\partial}{\partial t}\right)\left(\frac{\partial}{\partial t}+\tau_{q} \frac{\partial^{2}}{\partial t^{2}}\right) \frac{\partial^{2} w}{\partial x^{2}} \\
=\left(\frac{\partial}{\partial t}+\tau_{q} \frac{\partial^{2}}{\partial t^{2}}\right)\left(\eta+2 \Delta_{E} \frac{(1+v)}{(1-2 v)}\left(1+E_{1} \frac{\partial}{\partial t}\right)\right) \theta .
\end{gathered}
$$

We consider the following functions:

$$
\begin{aligned}
w(x, t) & =W(x) e^{i \omega t}, \\
\theta(x, y, t) & =\Theta(x, y) e^{i \omega t} .
\end{aligned}
$$

Hence, equation (17) will be in the form

$$
\begin{aligned}
(1 & \left.+i \tau_{T} \omega\right) \frac{\partial^{2} \Theta}{\partial y^{2}}+\frac{\Delta_{E}}{\alpha_{T}} y\left(1+i E_{1} \omega\right)\left(i \omega-\tau_{q} \omega^{2}\right) \frac{\mathrm{d}^{2} W}{\mathrm{~d} x^{2}} \\
= & \left(i \omega-\tau_{q} \omega^{2}\right)\left(\eta+2 \Delta_{E} \frac{(1+v)}{(1-2 v)}\left(1+i E_{1} \omega\right)\right) \Theta,
\end{aligned}
$$

which gives

$$
\left(\frac{\partial^{2}}{\partial y^{2}}-\lambda^{2}\right) \Theta(x, y)=-\alpha y \frac{\mathrm{d}^{2} W(x)}{\mathrm{d} x^{2}},
$$

where

$$
\begin{aligned}
& \lambda=\lambda(\omega)=\sqrt{\frac{\left(i \omega-\tau_{q} \omega^{2}\right)}{\left(1+i \tau_{T} \omega\right)}\left(\eta+2 \Delta_{E} \frac{(1+v)}{(1-2 v)}\left(1+i E_{1} \omega\right)\right)}, \\
& \alpha=\alpha(\omega)=\frac{\Delta_{E}}{\alpha_{T}} \frac{\left(1+i E_{1} \omega\right)\left(i \omega-\tau_{q} \omega^{2}\right)}{\left(1+i \tau_{T} \omega\right)} .
\end{aligned}
$$

The general solution of the differential equation (20) takes the form

$$
\Theta(x, y)=A \cosh (\lambda y)+B \sinh (\lambda y)+\frac{\alpha}{\lambda^{2}} y \frac{\mathrm{d}^{2} W(x)}{\mathrm{d} x^{2}} .
$$

The boundary conditions are as follows:

$$
\left.\frac{\partial \Theta(x, y)}{\partial y}\right|_{y=0}=\left.\frac{\partial \Theta(x, y)}{\partial y}\right|_{y=h}=0 .
$$

Hence, we obtain

$$
\Theta=\left[y-\frac{\sinh (\lambda y)}{\lambda \cosh ((\lambda h / 2))}\right] \frac{\alpha}{\lambda^{2}} \frac{\mathrm{d}^{2} W}{\mathrm{~d} x^{2}} .
$$

From equations (6), (8), and (18), we obtain

$$
\begin{aligned}
& \frac{E_{0}\left(1+i \omega E_{1}\right) I}{\rho A} \frac{\partial^{4} W(x)}{\partial x^{4}}+\frac{E_{0}\left(1+i \omega E_{1}\right) \alpha_{T}}{\rho A} \frac{\partial^{2}}{\partial x^{2}} \\
& \cdot \int_{(-b / 2)}^{(b / 2)} \int_{(-h / 2)}^{(h / 2)} y \Theta(x, y) d y d z-\frac{F}{\rho A} \frac{\partial^{2} W(x)}{\partial x^{2}}=\omega^{2} W(x) .
\end{aligned}
$$

Substituting from equation (24) in equation (25), we obtain

$$
\begin{aligned}
& \frac{E_{0}\left(1+i \omega E_{1}\right) I}{\rho A} \frac{\partial^{4} W(x)}{\partial x^{4}}+\frac{E_{0}\left(1+i \omega E_{1}\right) \alpha_{T} \alpha}{\rho A \lambda^{2}} \frac{\partial^{4} W(x)}{\partial x^{4}} \int_{(-b / 2)}^{(b / 2)} \\
& \cdot \int_{(-h / 2)}^{(h / 2)} y\left[y-\frac{\sin (\lambda y)}{\lambda \cos (\lambda h / 2)}\right] \mathrm{d} y \mathrm{~d} z-\frac{F}{\rho A} \frac{\partial^{2} W(x)}{\partial x^{2}}=\omega^{2} W(x) .
\end{aligned}
$$

Finally, we obtain

$$
\frac{I E_{\omega}}{\rho A}\left(\frac{\partial^{4} W(x)}{\partial x^{4}}-\frac{F}{I E_{\omega}} \frac{\partial^{2} W(x)}{\partial x^{2}}\right)=\omega^{2} W(x),
$$

where $E_{\omega}=E_{0}\left(1+i \omega E_{1}\right) f(\omega)$ and $f(\omega)=1+\left(\alpha_{T} b \alpha / \lambda\right.$ $\left.{ }^{2} I\right)\left(\left(h^{3} / 12\right)+\left(h / \lambda^{2}\right)-\left(2 / \lambda^{3}\right) \tan ((\lambda / 2))\right)$.

For a simply supported beam, the exact analytical solution for the natural frequency is given as [28]

$$
\omega=\frac{\pi}{L \sqrt{\rho A}} \sqrt{\frac{\pi^{2} I E_{0}}{L^{2}}\left(1+i \omega_{0} E_{1}\right) f\left(\omega_{0}\right)+F},
$$

where $\omega_{0}$ is the isothermal value of fundamental frequency given by $[10,30]$

$$
\omega_{0}=q^{2} \frac{b}{L^{2}} \sqrt{\frac{E_{0}}{12 \rho}} .
$$

For beams with both ends clamped, $q=4.73$, while $q=\pi$ for beams with both ends simply support.

Hence, we obtain 


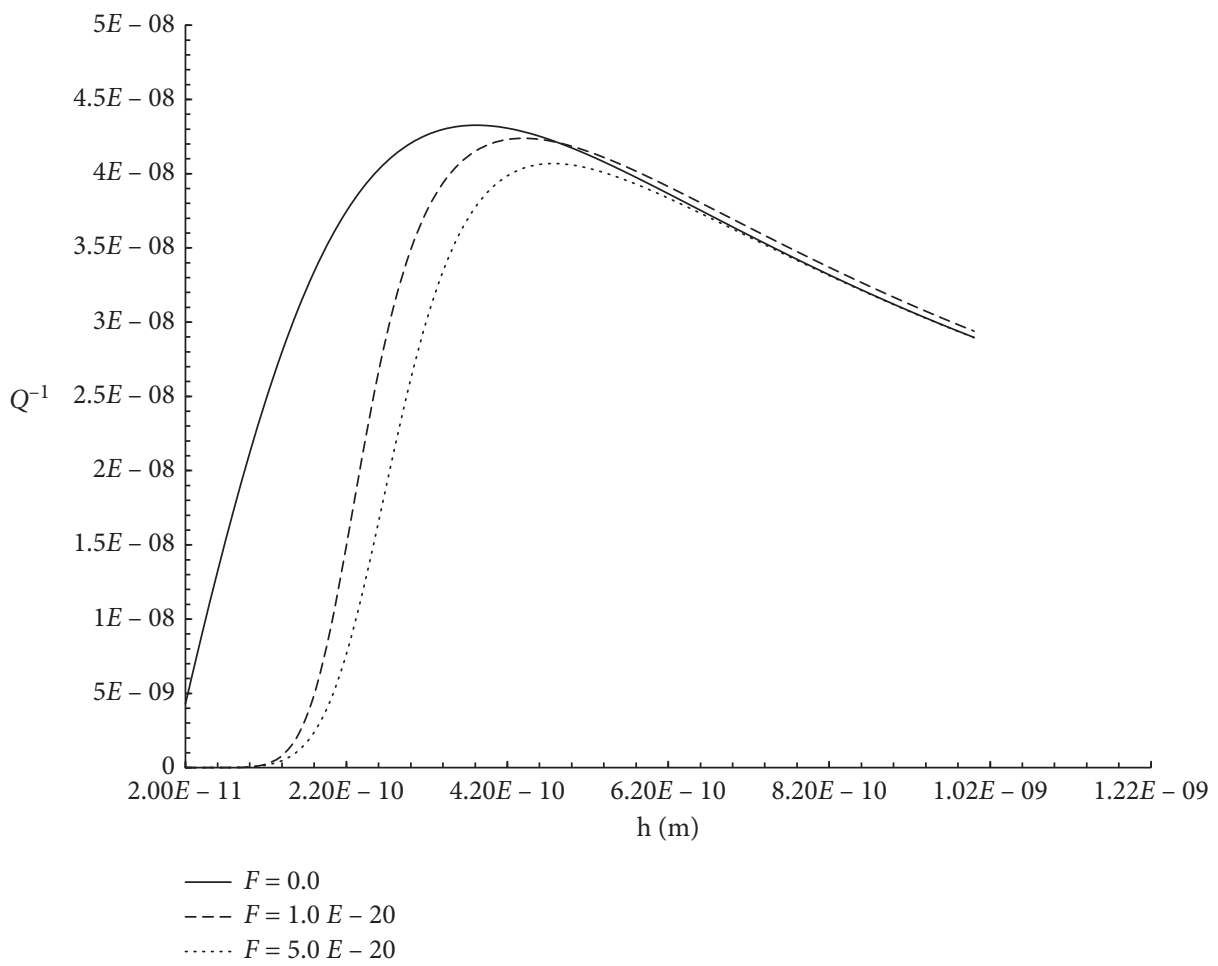

FIGURE 1: The quality factor of the resonator with a different value of static pre-stress.

$$
\omega=\frac{\pi^{2}}{L^{2}} \sqrt{\frac{I E_{0}}{\rho A}} \sqrt{\left(1+i E_{1} \omega_{0}\right) 1+\frac{\Delta E \operatorname{Eh}^{3}\left(1+i E_{1} \omega_{0}\right)}{12 I \varepsilon_{1}} 1+\left(\frac{12 \varepsilon 2}{h^{2} \varepsilon 1}-\frac{24}{h^{3}} \tanh \left(\frac{\lambda h}{2}\right)\left(\frac{\varepsilon_{1}}{1+i \tau_{T} \omega 0}\right)^{-(3 / 2)}\right)}+\frac{L^{2}}{\pi^{2} I^{2} E_{0}} F
$$

where $\varepsilon_{1}=\eta+\left(2 \Delta E(1+v)\left(1+i E_{1} \omega_{0}\right) / 1-2 v\right)$ and $\varepsilon_{2}=$ $\left(1+i \tau_{T} \omega_{0} / i \omega_{0}-\tau_{q} \omega_{0}^{2}\right)$

The quality factor $\left(Q^{-1}\right)$ is defined as follows:

$$
Q^{-1}=2\left|\frac{\operatorname{Im}(\omega)}{\operatorname{Re}(\omega)}\right| \text {. }
$$

\section{Numerical Results and Discussion}

Now, the relationships between the variation of the thermoelastic damping with the beam height $h$, and the beam length in different values of the applied axial force $F$ on the microbeam resonator, which is made of silicon and clamped at two ends, will be explored.

Material properties of silicon $(\mathrm{Si})$ have been taken as follows $[10,30]$ :

$T_{0}=300(K), \quad \rho=2330\left(\mathrm{kgm}^{-3}\right), K=141.04\left(\mathrm{Wm}^{-1}\right.$ $\left.k^{-1}\right), C_{v}=1.64 \times 10^{6}\left(\mathrm{Jm}^{-3} K^{-1}\right), E=165(\mathrm{GPa})$, and $\alpha=$ $2.6 \times 10^{-6}\left(K^{-1}\right)$. The aspect ratios of the beam are fixed as $(L / h)=50$ and $b=5 h$. For the micro-scale beam, we will take the beam's thickness $h\left(10^{-11} m \leq h \leq 10^{-9} \mathrm{~m}\right)$.

Figure 1 represents the quality factor of the resonator with a different value of the force $F=(0.0,1.0,5.0) \times$ $10^{-20} \mathrm{Kgms}^{-2}$ of the static pre-stress. It is noted that the force due to the static pre-stress has a significant effect on the quality factor $\left(Q^{-1}\right)$. The value of the quality factor $\left(Q^{-1}\right)$ decreases when the value of static pre-stress increases until the position $h=6.0 \times 10^{-10} \mathrm{~m}$; then, the three cases are very closed. In Figure 1, for the case $F=0.0 \mathrm{Kgms}^{-1}$, the curve has the same attitude of the curves of Figure 3 in [30], Figure 2 in [31], and Figure 8 in [32].

Figure 2 represents the inverse of the quality factor $\left(Q^{-1}\right)$ of the resonator with a different value of the mechanical relaxation time $E_{1}=(0.0,10.0,20.0) \times 10^{-22} s$ of the static pre-stress. It is noted that the value of mechanical relaxation time has a significant effect on the quality factor. In essence, the value of the inverse of the quality factor $\left(Q^{-1}\right)$ decreases when the value of the mechanical relaxation time increases. In Figure 2, for the case $E_{1}=0.0 \mathrm{~s}$, the curve agrees with the curves of Figure 3 in [30], Figure 2 in [31], and Figure 8 in [32].

Figure 3 represents the quality factor $\left(Q^{-1}\right)$ of the resonator with a different value of the isothermal value of frequency $\omega_{0}(q)$, where $q=(\pi, 4.73,7.85)$. It is noted that the value of the isothermal value of frequency has a significant effect on the quality factor $\left(Q^{-1}\right)$ of the thermal damping; the value of the quality factor $\left(Q^{-1}\right)$ increases when the isothermal value of frequency decreases.

Figure 4 represents the inverse of the quality factor $\left(Q^{-1}\right)$ of the resonator with a different value of the ratio of the thermal relaxation times $\tau_{q} / \tau_{T}=(20,25,30)$, respectively. It is noted that the values of the ratio of the thermal 


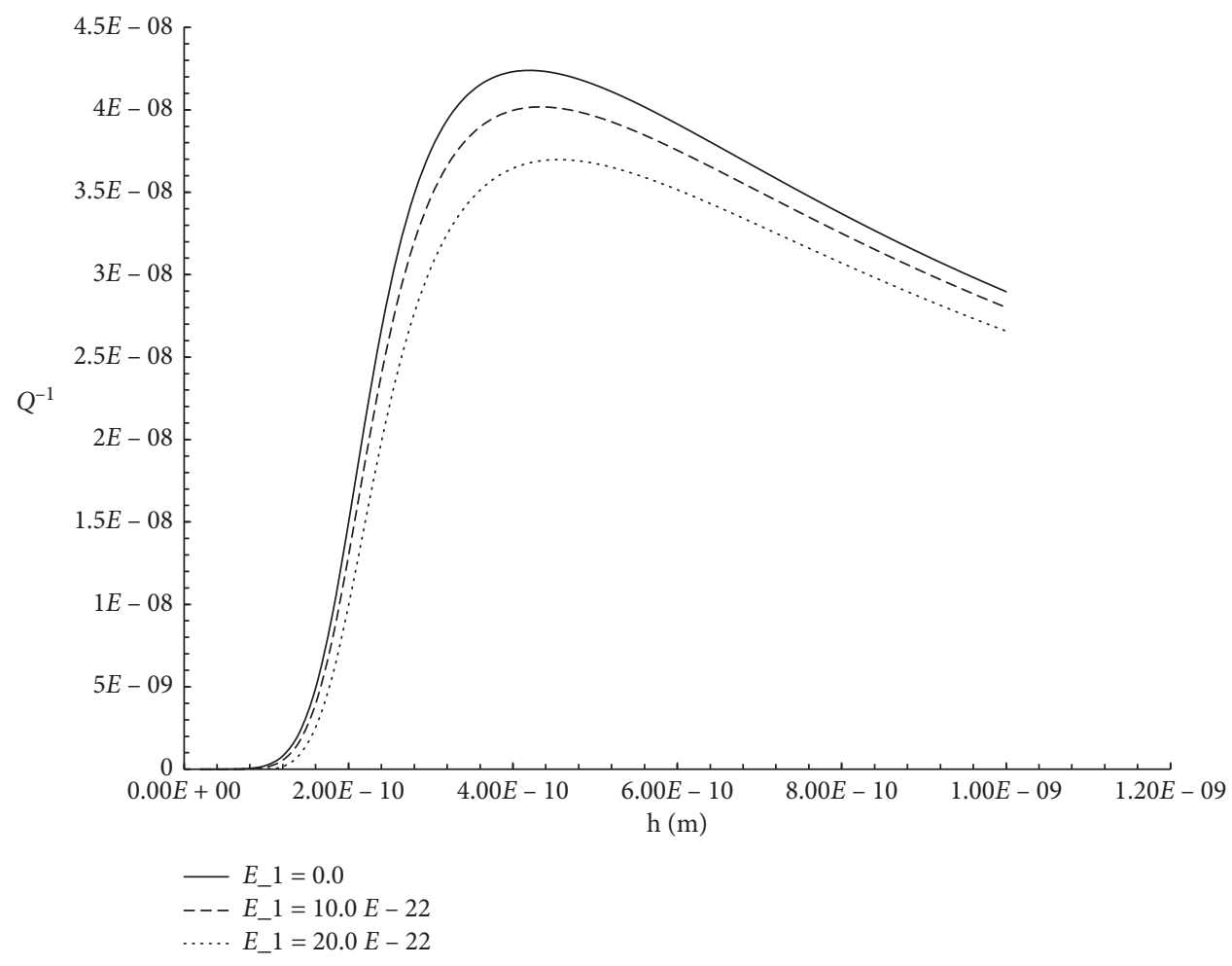

Figure 2: The quality factor of the resonator with a different value of mechanical relaxation time parameter.

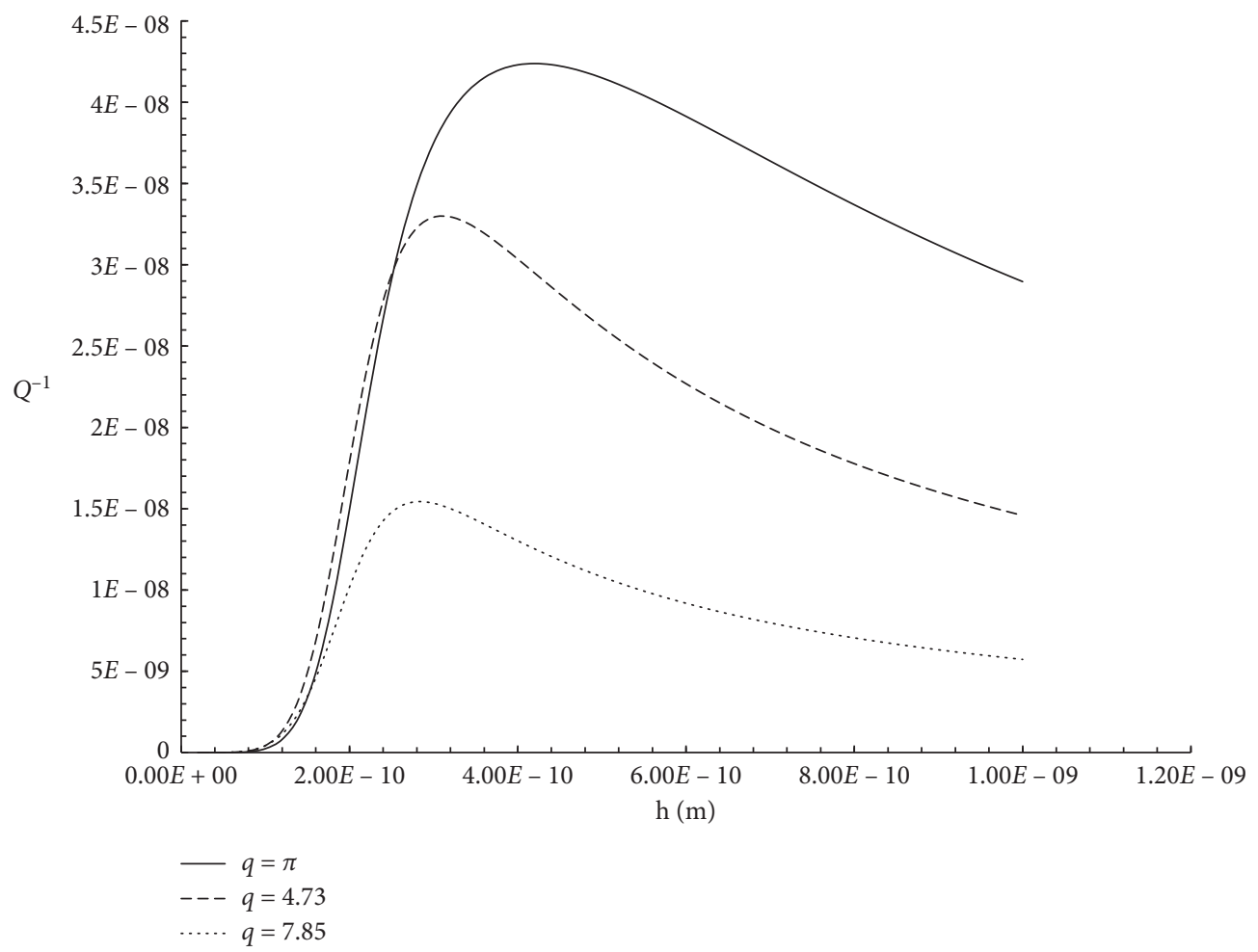

Figure 3: The quality factor of the resonator with a different value of the isothermal value of frequency $\omega_{0}\left(q_{n}\right)$. 


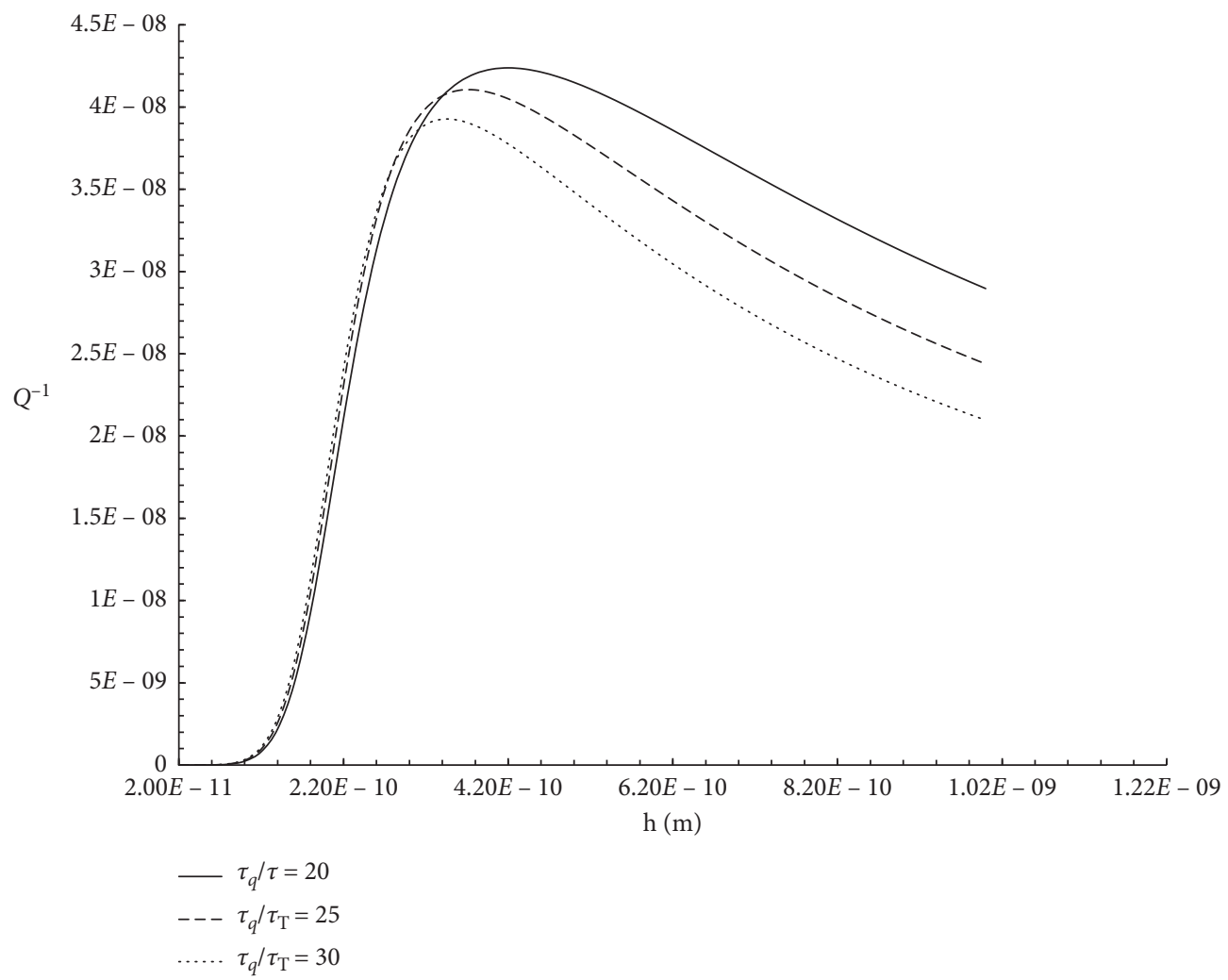

FIgURE 4: The quality factor of the resonator with a different ratio of thermal relaxation times.

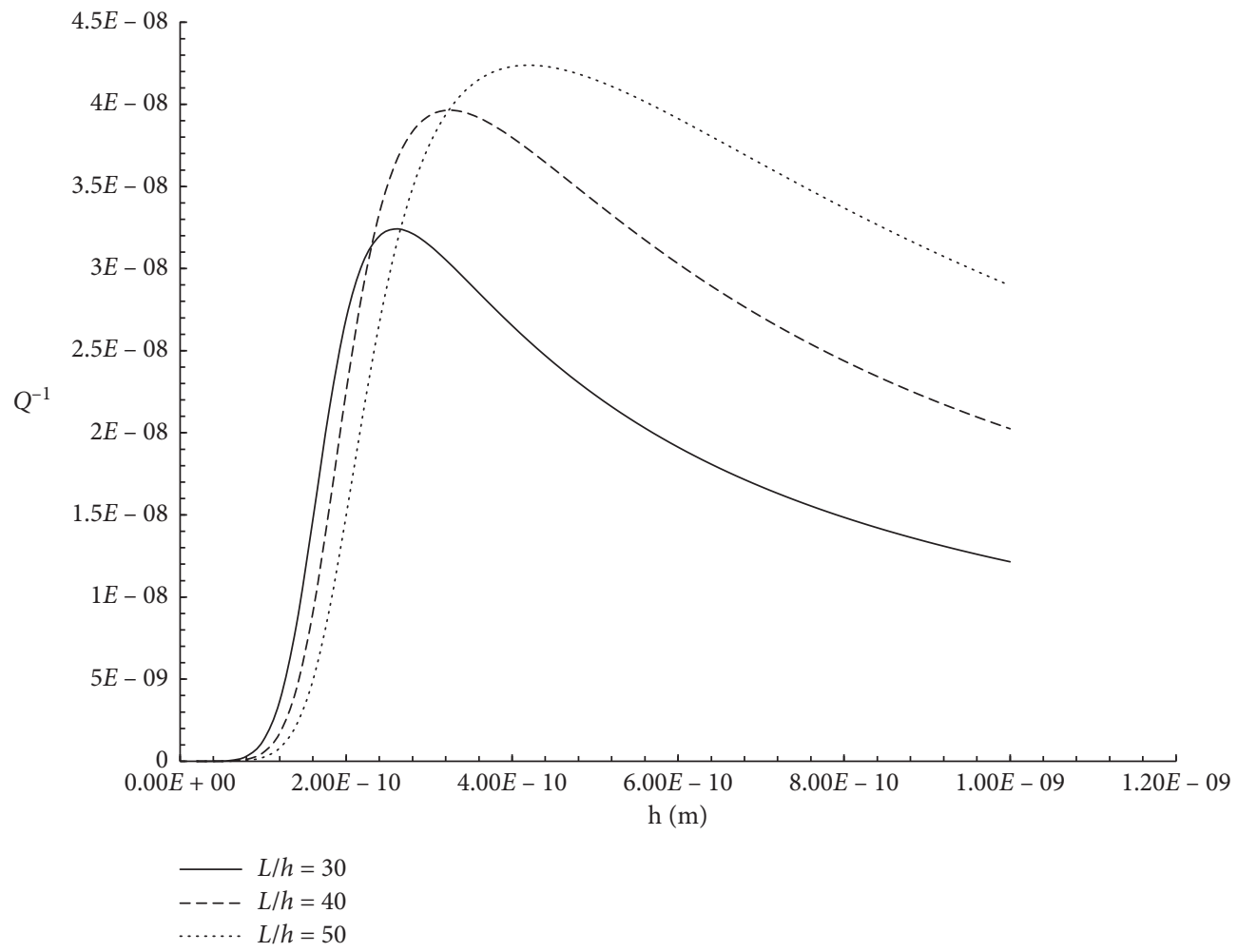

FIGURE 5: The quality factor of the resonator with a different value of size ratio $L / h$. 
relaxation times have a significant effect on the quality factor $\left(Q^{-1}\right)$ of the thermal damping. The value of the quality factor $\left(Q^{-1}\right)$ decreases when the value of the ratio $\tau_{q} / \tau_{T}$ increases. In Figure 4, the curves agree with the curves of Figure 3 in [30], Figure 2 in [31], and Figure 8 in [32].

Figure 5 represents the quality factor $\left(Q^{-1}\right)$ of the resonator with a different value of the size ratio $(L / h)=(30,40,50)$, respectively. It is noted that the values of the size ratio have a significant effect on the quality factor $\left(Q^{-1}\right)$; the value of the quality factor $\left(Q^{-1}\right)$ increases when the value of the size ratio $L / h$ increases.

\section{Conclusion}

An analytical model for viscothermoelastic relaxation times in flexural resonators under axial pre-stress has been developed in the micro-scale. The model confirms that a significant reduction in the quality factor as a tensile axial pre-stress is applied. This reduction in damping can be correlated with a decrease in the relaxation rate as compared to the natural frequency of the resonator. The force due to the static pre-stress, isothermal frequency, thermal relaxation times, mechanical relaxation time, and size ratio have a significant effect on the quality factor of the nanobeams.

\section{Data Availability}

No data were used to support this study.

\section{Conflicts of Interest}

The authors declare that there are no conflicts of interest in this work.

\section{References}

[1] A. Duwel, R. N. Candler, T. W. Kenny, and M. Varghese, "Engineering MEMS resonators with low thermoelastic damping," Journal of Microelectromechanical Systems, vol. 15, no. 6, pp. 1437-1445, 2006.

[2] F. L. Guo and G. A. Rogerson, "Thermoelastic coupling effect on a micro-machined beam resonator," Mechanics Research Communications, vol. 30, no. 6, pp. 513-518, 2003.

[3] C. M. Harris and A. G. Piersol, Harris' Shock and Vibration Handbook, McGraw-Hill, New York, NY, USA, 2002.

[4] T. Koyama, D. S. Bindel, W. He et al., Simulation Tools for Damping in High Frequency Resonators, IEEE, in Proceedings of the SENSORS, 2005, p. 4, IEEE, Irvine, CA, USA, October 2005.

[5] C. Zener, "Internal friction in solids. I. Theory of internal friction in reeds," Physical Review, vol. 52, no. 3, pp. 230-235, 1937.

[6] C. Zener, "Internal friction in solids II. General theory of thermoelastic internal friction," Physical Review, vol. 53, no. 1, pp. 90-99, 1938.

[7] C. Zener, W. Otis, and R. Nuckolls, "Internal friction in solids III. Experimental demonstration of thermoelastic internal friction," Physical Review, vol. 53, no. 1, pp. 100-101, 1938.

[8] H. M. Youssef and M. W. Al-Hazmi, "The influence of the static-pre-stress and mechanical damage variable in the thermal quality factor of two-temperature viscothermoelastic resonators," Advances in Mechanical Engineering, vol. 12, no. 6, Article ID 1687814020930454, 2020.

[9] R. Lifshitz and M. L. Roukes, "Thermoelastic damping in micro- and nanomechanical systems," Physical Review B, vol. 61 , no. 8 , pp. 5600-5609, 2000.

[10] Y. Sun, D. Fang, and A. K. Soh, "Thermoelastic damping in micro-beam resonators," International Journal of Solids and Structures, vol. 43, no. 10, pp. 3213-3229, 2006.

[11] J. N. Sharma and R. Sharma, "Damping in micro-scale generalized thermoelastic circular plate resonators," Ultrasonics, vol. 51, no. 3, pp. 352-358, 2011.

[12] D. Tzou, "On the thermal shock wave induced by a moving heat source," Journal Heat Transfer, vol. 112, 1989.

[13] D. Tzou, Transfer, the Lagging Behavior, Taylor \& Francis, Washington, DC, USA, 1997.

[14] M. Xu, J. Guo, L. Wang, and L. Cheng, "Thermal wave interference as the origin of the overshooting phenomenon in dual-phase-lagging heat conduction," International Journal of Thermal Sciences, vol. 50, no. 5, pp. 825-830, 2011.

[15] N. S. Al-Huniti and M. A. Al-Nimr, "Thermoelastic behavior of a composite slab under a rapid dual-phase-lag heating," Journal of Thermal Stresses, vol. 27, no. 7, pp. 607-623, 2004.

[16] J.-R. Ho, C.-P. Kuo, and W.-S. Jiaung, "Study of heat transfer in multilayered structure within the framework of dual-phaselag heat conduction model using lattice Boltzmann method," International Journal of Heat and Mass Transfer, vol. 46, no. 1, pp. 55-69, 2003.

[17] Y.-M. Lee and T.-W. Tsai, "Ultra-fast pulse-laser heating on a two-layered semi-infinite material with interfacial contact conductance," International Communications in Heat and Mass Transfer, vol. 34, no. 1, pp. 45-51, 2007.

[18] K.-C. Liu, "Numerical analysis of dual-phase-lag heat transfer in a layered cylinder with nonlinear interface boundary conditions," Computer Physics Communications, vol. 177, no. 3, pp. 307-314, 2007.

[19] K. Ramadan, "Semi-analytical solutions for the dual phase lag heat conduction in multilayered media," International Journal of Thermal Sciences, vol. 48, no. 1, pp. 14-25, 2009.

[20] N. Alghamdi, "Dual-phase-Lagging thermoelastic damping vibration in micro-nano scale beam resonators with voids," International Journal of Multidisciplinary and Current Research, vol. 5, 2017.

[21] N. A. Alghamdi and H. M. Youssef, "Dual-phase-lagging thermoelastic damping in-extensional vibration of rotating nano-ring," Microsystem Technologies, vol. 23, no. 10, pp. 4333-4343, 2017.

[22] F. L. Guo, J. Song, G. Q. Wang, and Y. F. Zhou, "Analysis of thermoelastic dissipation in circular micro-plate resonators using the generalized thermoelasticity theory of dual-phaselagging model," Journal of Sound and Vibration, vol. 333, no. 11, pp. 2465-2474, 2014.

[23] F. L. Guo, G. Q. Wang, and G. A. Rogerson, "Analysis of thermoelastic damping in micro- and nanomechanical resonators based on dual-phase-lagging generalized thermoelasticity theory," International Journal of Engineering Science, vol. 60, pp. 59-65, 2012.

[24] M. A. Biot, "Theory of Stress-Strain Relations in Anisotropic Viscoelasticity and Relaxation Phenomena," Journal of Applied Physics, vol. 25, no. 11, pp. 1385-1391, 1954.

[25] M. A. Biot, "Variational principles in irreversible thermodynamics with application to viscoelasticity," Physical Review, vol. 97, no. 6, pp. 1463-1469, 1955. 
[26] A. Drozdov, "A constitutive model in finite thermoviscoelasticity based on the concept of transient networks," Acta Mechanica, vol. 133, no. 1-4, pp. 13-37, 1999.

[27] M. A. Ezzat and A. S. El-Karamany, "The relaxation effects of the volume properties of viscoelastic material in generalized thermoelasticity," International Journal of Engineering Science, vol. 41, no. 19, pp. 2281-2298, 2003.

[28] F. J. Shaker, Effect of Axial Load on Mode Shapes and Frequencies of Beams, National Aeronautics and Space Administration, Washington, DC, USA, 1975.

[29] S. S. Verbridge, D. F. Shapiro, H. G. Craighead, and J. M. Parpia, "Macroscopic tuning of nanomechanics: substrate bending for reversible control of frequency and quality factor of nanostring resonators," Nano Letters, vol. 7, no. 6, pp. 1728-1735, 2007.

[30] H. M. Youssef and N. A. Alghamdi, "Thermoelastic damping in nanomechanical resonators based on two-temperature generalized thermoelasticity theory," Journal of Thermal Stresses, vol. 38, no. 12, pp. 1345-1359, 2015.

[31] H. Kumar and S. Mukhopadhyay, "Analysis of the quality factor of micro-beam resonators based on heat conduction model with a single delay term," Journal of Thermal Stresses, vol. 42, no. 8, pp. 929-942, 2019.

[32] E. K. Kakhki, S. M. Hosseini, and M. Tahani, "An analytical solution for thermoelastic damping in a micro-beam based on generalized theory of thermoelasticity and modified couple stress theory," Applied Mathematical Modelling, vol. 40, no. 4, pp. 3164-3174, 2016. 